\title{
Application of the small punch test to determine the fatigue properties of additive manufactured aerospace alloys
}

\author{
Robert Lancaster ${ }^{1, *}$, Henry Illsley ${ }^{1}$, Spencer Jeffs ${ }^{1}$, Roger Hurst $^{1}$ and Gavin Baxter ${ }^{2}$ \\ ${ }^{1}$ Swansea University, Institute of Structural Materials, Bay Campus, Fabian Way, Swansea, SA1 8EN, United Kingdom \\ ${ }^{2}$ Rolls-Royce plc, PO Box 31, Derby, DE24 8BJ, United Kingdom
}

\begin{abstract}
Additive layer manufacturing (ALM) processes are becoming increasingly prevalent in the aerospace industry as design engineers look to profit from the numerous advantages that these advanced techniques can offer. However, given the safety critical nature and arduous operating conditions to which these components will be exposed to whilst in service, it is essential that the mechanical properties of such structures are fully understood. Transient microstructures are a typical characteristic of ALM components and resulting from the thermal cycles that occur during the build operation. Those microstructures make any mechanical assessment an involved procedure when assessing the process variables for any given parameter set. A useful mechanical test technique is small-scale testing, in particular, the small punch (SP) test. SP testing is capable of localised sampling of a larger scale component and presents an attractive option to mechanically assess complex parts with representative geometries, that would not be possible using more conventional uniaxial test approaches. This paper will present the recent development of a small-scale testing methodology capable of inducing fatigue damage and a series of novel tests performed on different variants of Ti$6 \mathrm{Al}-4 \mathrm{~V}$.
\end{abstract}

\section{Introduction}

Additive layer manufacturing (ALM) is a rapidly growing technology receiving widespread attention from numerous industrial sectors. The process has evolved significantly over recent years and is capable of fabricating near net shape, fully dense structural parts with a high potential for geometrical optimisation. The emergence of ALM is linked to the significant benefits that the process can offer compared to more conventional manufacturing processes, such as forging or casting. These include considerable cost savings due to less material wastage, short lead times and improved buy-tofly ratios in the gas turbine industry, whilst also being capable of forming highly intricate components that would not be possible with more traditional methods.

One such ALM technique is electron beam melting (EBM), a powder bed process where an electron beam melts metal powder on a retractable base plate to build a three-dimensional component layer by layer, typically within a near vacuum environment. Due to the inert atmosphere, materials which have a high affinity towards oxygen, such as titanium alloys, can be readily manufactured using this process Previous research details the various aspects of EBM Ti alloys, particularly Ti-6AI$4 \mathrm{~V}$, including the presence of porosity, build artefacts, optimal parameter sets and powder characteristics [1], [2]. However, understanding the transient microstructures which are typically produced in an EBM build material, that can in turn lead to varying the mechanical properties across the component, is a fundamental research requirement. Furthermore, considering the multiple interactions of intra and inter build process variables on the integrity and consistency of the final structure, traditional laboratory scale test approaches are deemed unsuitable for mechanical characterisation as it is difficult to extract representative test specimens which comply to relevant standards. As such, small scale testing can offer a pragmatic alternative for initial assessment and verification.

Previous research has revealed that small scale mechanical testing approaches, in particular the small punch (SP) test, are now widely regarded as an established means of obtaining useful mechanical property information from limited material quantities To date these methods have largely been adopted to characterise the creep [3], [4], tensile [5], [6] and fracture characteristics [7], [8] of numerous material systems [9], [10] from a range of industrial applications for sampling [11], [12]. This paper will now detail the research undertaken in realising a small-scale test methodology capable of characterising the localised fatigue properties of ALM built structures A series of novel experimentsare presented on Ti-6AI-4V materials fabricated through two different manufacturing processes.

\section{Experimental methods}

\subsection{Materials}

For this research, the study was carried out on two Ti-6Al4V variants, manufactured using different processing routes: (i) a forged disk and (ii) Electron Beam Melting

\footnotetext{
* Corresponding author: r.j.lancaster@swansea.ac.uk
} 
(EBM). The EBM material was built in a vertical orientation on an Arcam System configured with EBM Control 3.2 Service Pack software. The pre-alloyed plasma atomised Ti-6Al-4V powder feedstock contained spherical particles ranging from $45-100 \mu \mathrm{m}$ in diameter. The first layer of powder was deposited onto a $220 \times 300$ $x 10 \mathrm{~mm}^{3}$ thick stainless steel base plate that was preheated with the electron beam to approximately $730^{\circ} \mathrm{C}$. Prior to melting each layer, the new layer of powder was preheated and sintered by scanning the beam over the cross-sectional geometry in an attempt to maintain a consistent build temperature of $730^{\circ} \mathrm{C}$ throughout and reduce the effects of charging of the powder during melting. Further information on this process can be found in [13], [14].

Figures 1 and 2 illustrate the microstructures for the two materials. The EBM material is shown in both the face parallel to the build direction and the face perpendicular to the build direction, highlighting the anisotropy in the material. When looking into the build direction, or the SP specimen surface, (Figure 1a, b) the microstructure displays an ordered lamellar structure consisting of an extremely fine acicular grain morphology. This results from the thermal characteristics of the EBM process where a small melt pool and rapid cooling results in a columnar prior- $\beta$ grain structure with epitaxial $\beta<100>$ crystal growth parallel to the build direction (Figure 1c, d). Here, the elongated grain widths were measured perpendicular to the length of the grain using the mean linear intercept (MLI) method. The forged variant (Figure 2) has an equiaxed bimodal structure, with an $\alpha$ volume fraction of approximately $60 \%$.

Additional microstructural measurements characterising the typical grain sizes of the different Ti-6Al-4V variants are given in Table 1 . In each material, the measurements are taken perpendicular to the textured grain's largest axis and represent an average from over 600 calculations from multiple locations within the sample.

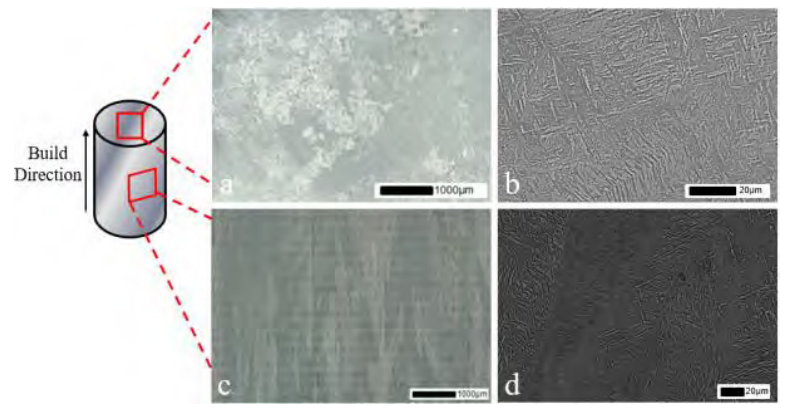

Figure 1. Microstructure of EBM Ti-6AI-4V (a) perpendicular to the build direction at low magnification; (b) perpendicular to the build direction at high magnification; (c) parallel to the build direction at low magnification and (d) parallel to the build direction at high magnification. The force is applied through the face perpendicular to the build direction.

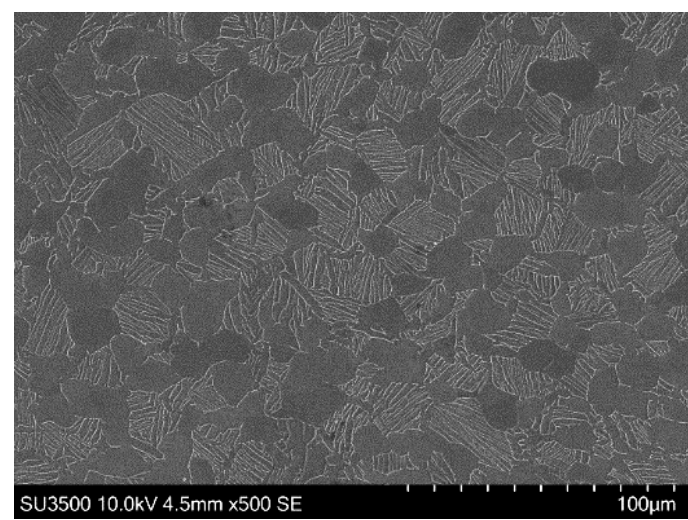

Figure 2. Microstructure of forged Ti-6Al-4V, illustrating the top surface of the SP specimen where the punch makes contact.

Table 1. Microstructural measurements of the two Ti-6AI-4V variants

\begin{tabular}{|l|l|l|}
\hline Measurement & Forged & EBM \\
\hline Mean Linear Intercept $(1 / \mu \mathrm{m})$ & 0.0778 & 0.0041 \\
\hline Prior- $\beta$ grain width $(\mu \mathrm{m})$ & 13 & 246 \\
\hline Transformed alpha width $(\mu \mathrm{m})$ & 1.6868 & 0.7867 \\
\hline
\end{tabular}

\subsection{Small punch specimens}

SP specimens were extracted from the threaded stub ends of conventional tensile specimens for the two materials and were then turned down to a diameter of $9.5 \mathrm{~mm}$. The resulting cylinders were then sectioned into slices approximately $800 \mu \mathrm{m}$ in thickness before being ground down to the required thickness of $500 \mu \mathrm{m} \pm 5 \mu \mathrm{m}$ using successively finer grades of silicon carbide abrasive paper, the final grade being 1200 grit in accordance with the recommendations of the European Code of Practice for Small Punch testing (EUCoP) [15]. The thickness was then measured at 5 locations around the periphery and the centre of the specimen to ensure uniformity.

\subsection{Experimental set up}

A bespoke SP fatigue test capability has been developed at Swansea University to test miniature disc specimens at both ambient and elevated temperatures under relatively low loading conditions. The apparatus has been designed to be inserted into the load train of a universal, servoactuated test machine, with a load capacity of $\pm 5 \mathrm{kN}$ to ensure intimate control of the applied load. The test configuration consists of two Nimonic-90 punch indenters, one of which is positioned above the SP disc and the other located directly beneath. The two indenters maintain contact with the specimen throughout the test through a small residual force, which is introduced to eradicate any potential air gaps in the load train which may occur if the centre of the specimen begins to thin. The disc then vertically oscillates in movement to apply a fully reversed force to the material, replicating a fully reversed fatigue loading ratio of $\mathrm{R}=-1$. To monitor the evolving deformation, a linear variable displacement transducer (LVDT) measures the displacement of the 
disc. These values are recorded in addition to the position and force measurements from the test machine.

In the test set up, as presented in Figure 3, the miniature disc sample is clamped within an upper and lower die which is positioned within an upper housing along with the upper indenter which is located above the specimen. The lower indenter is placed within the lower pull rod and is slowly raised until it makes contact with the bottom surface of the disc, from which it is then held in a stationary position under zero force.

For both the upper and lower indenters, the applied force is transferred to the specimen via a $2 \mathrm{~mm}$ diameter hemispherical ended Nimonic-90 punch, perpendicular to the top and bottom surfaces of the disc, through a receiving hole of $4 \mathrm{~mm}$ diameter with a $0.2 \mathrm{~mm}$ radius chamfer. These dimensions are in direct compliance to the directives detailed in the European $\mathrm{CoP}$ [15]. A schematic representation of this arrangement is displayed in Figure 3.

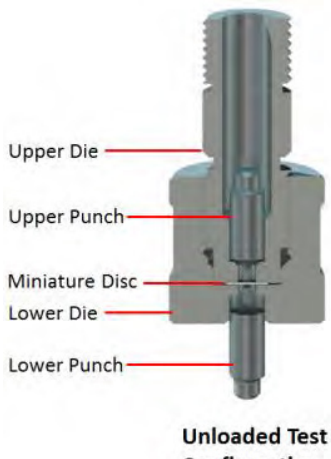

Configuration
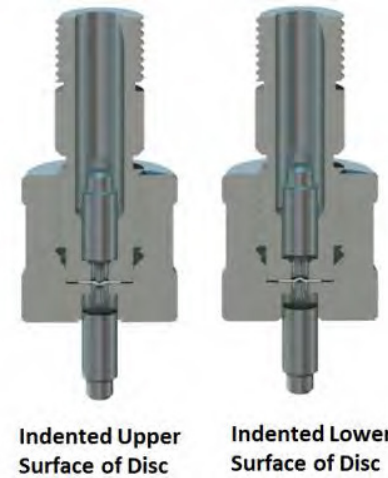

Indented Lower Surface of Disc
Figure 3. Representation of the small punch fatigue test configuration.

\section{Results \& Discussion}

\subsection{Small punch fatigue results}

A series of SP fatigue tests were performed under various applied load ranges to generate representative force $(\mathrm{F})$ number of fatigue cycles to failure $(\mathrm{N})$ curves, or an F-N curve (Figure 4) for each of the materials. For each of the experiments, an $\mathrm{R}=-1$ waveform was adopted and was applied under a loading frequency of $1 \mathrm{~Hz}$ using a sinusoidal waveform. Each test was interrupted once a displacement range of $250 \mu \mathrm{m}$ was achieved, from which a clearly defined crack was identifiable. At this point, the disc was considered fractured and the test was terminated.

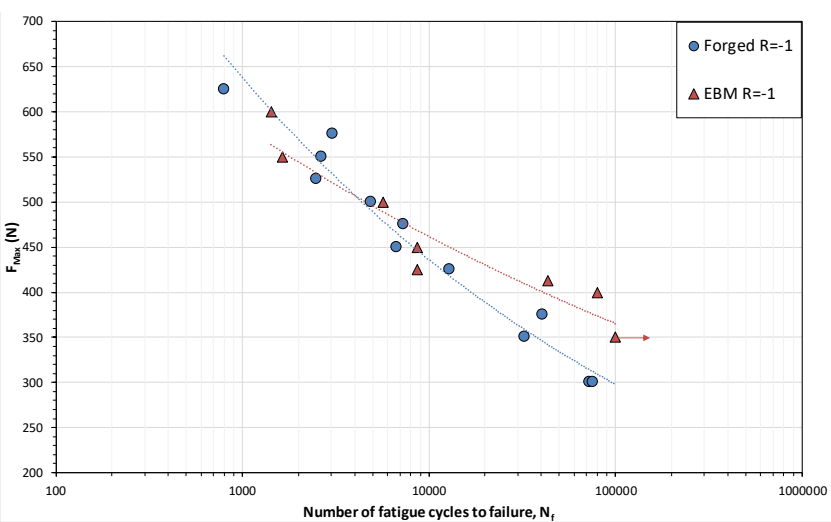

Figure 4. Small punch fatigue results $\left(20^{\circ} \mathrm{C}, \mathrm{R}=-1,1 \mathrm{~Hz}\right.$ sinusoidal waveform).

The behaviour of the two materials (as given in Figure 4) is highly comparable to the response that would typically be expected from a uniaxial stress $(\mathrm{S})$ - number of fatigue cycles to failure $(\mathrm{N})$ curve, in that as the force range applied to the specimen is increased, the number of cycles required to produce failure decreases. Even though the two test methods appear to produce a similar $\mathrm{S} / \mathrm{F}-\mathrm{N}$ response, the deformation behaviour is quite different. In a SP test, whether the load is applied to replicate tensile, creep or fatigue damage, the miniature disc sample is subjected to an initial period of compressive loading, followed by a bending motion and finally a stage of tensile elongation where the membrane deforms and thins, all of which occur under a transient biaxial stress condition. This is in contrast to a uniaxial arrangement where the specimen simply elongates or deforms under an applied tensile load, whether the load is static (as is the case for tensile or creep) or dynamic (fatigue). Therefore, biaxiality needs to be considered when attempting to correlate SP fatigue results with uniaxial data, particularly for anisotropic or heavily textured materials such as ALM parts.

Figure 5 shows the evolution of displacement in each of the SP fatigue tests on EBM Ti-6Al-4V and how the top and bottom surface displacement values behave during the duration of each.

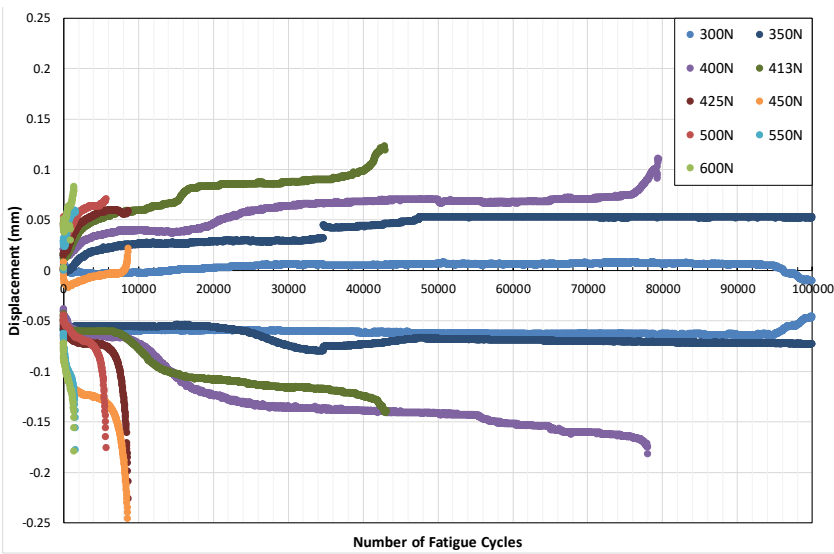

Figure 5. Evolution of upper and lower surface displacement behaviour for the small punch fatigue tests on EBM Ti-6Al-4V.

As can be seen, it appears that for the higher load tests (which produced the lowest lives), preferential deformation is more prevalent on the bottom surface of 
the disc, from the lower punch indenter penetrating upwards into the bottom surface of the material. This is a direct result of the experimental procedure itself, in which the load is initially exerted onto the lower punch and therefore, the bottom surface of the disc tends to deform at a slightly faster rate compared to the top surface. This pattern however, is not applicable for lower load tests since the magnitude of the applied load is not sufficiently high enough to introduce any significant plastic behaviour upon the initial loading cycles, similar to the yield strength when considering a uniaxial arrangement.

From these results, hysteresis force-displacement loops were generated for each test. Figure 6 presents the forcedisplacement loop responses for a SPF test performed on EBM Ti-6Al-4V under a maximum load of $400 \mathrm{~N}$. The figure shows the repeatability of the applied loading waveform through the entirety of the test where the maximum and minimum force values remain consistent throughout. Furthermore, the degree of plastic deformation, or displacement, in the disc can be seen to increase with an increasing number of fatigue cycles, replicating the expected behaviour of a component when subjected to a more traditional fatigue damage mechanism.

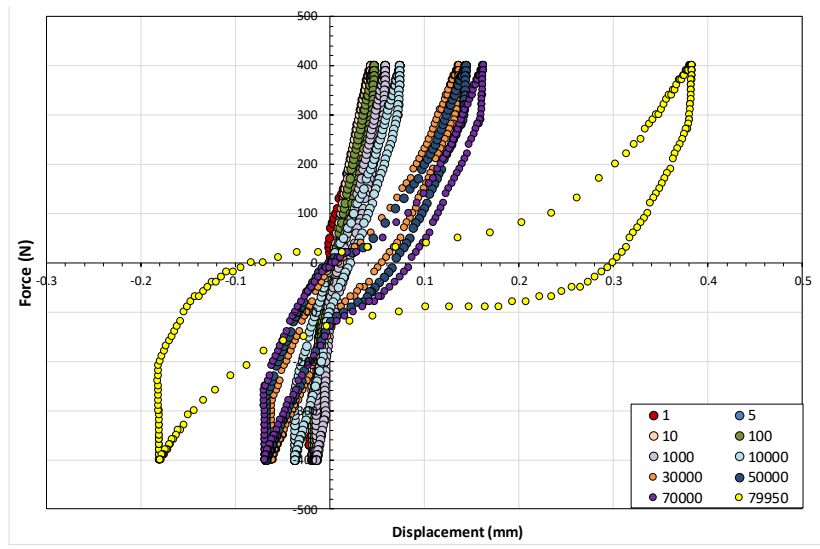

Figure 6. Hysteresis force-displacement loops for a SPF test performed on EBM Ti6Al-4V at a maximum force of $400 \mathrm{~N}$ $\left(20^{\circ} \mathrm{C}, \mathrm{R}=-1,1 \mathrm{~Hz}\right.$ sinusoidal waveform $)$.

\subsection{Fractography}

Supporting fractography was gathered for each of the tested specimens to develop an understanding of the contrasting manners of failure in the two materials. Figure 7 presents a direct comparison for a SPF test performed on each material under an applied $F_{\text {Max }}$ of $500 \mathrm{~N}$. The fracture behaviour in either case is quite similar, with a dominant 'star' type crack present, with the cracks propagating radially from a central location as the materials accumulate an increasing number of fatigue cycles, until the stretched membrane is unable to support the applied load and fractures.

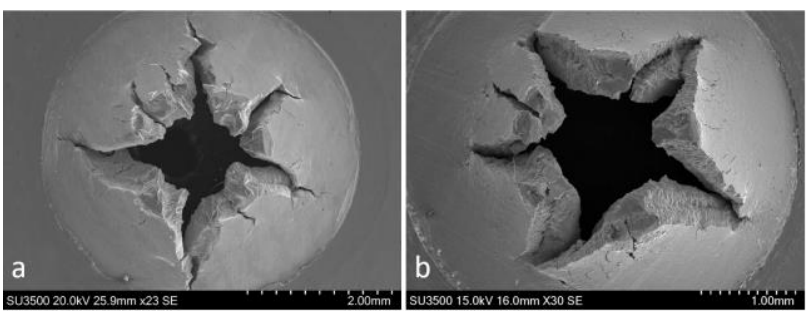

Figure 7. Low Magnification SEM Fracture surface images of (a) EBM and (b) Forged Ti-6Al-4V SPF tests performed at $\mathrm{F}_{\mathrm{Max}}=500 \mathrm{~N}$.

Additional fractography is provided in Figure 8, highlighting some of the prominent features on each of the fracture surfaces at a higher magnification.

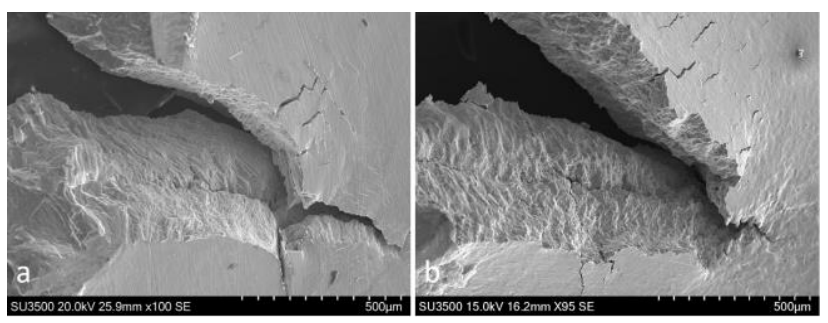

Figure 8. High Magnification SEM Fracture surface images of (a) EBM and (b) Forged Ti-6Al-4V SPF tests performed at $\mathrm{F}_{\mathrm{Max}}=500 \mathrm{~N}$.

In both materials, the fracture characteristics are very similar, with secondary cracking clearly observed along the length of the fractured segments, the extent of which is highly comparable between the two.

When considering fatigue crack growth, the fracture surface of the material is usually associated with the presence of striations, a feature that provides adequate evidence that the component was subjected to a form of cyclic loading. Striations typically represent a single increment of crack growth that occur within a loading cycle through the plastic blunting process. However, as is commonly found in uniaxial fatigue tests where a compressive loading ratio is employed, striations were not seen due to crack closure events as the disc cycles through zero load in each fatigue cycle. As such, any striations that may have been present in the radial cracks have been simply worn away by the crack opening-closing process.

\subsection{Crack path morphologies}

Following fractographic analysis, the failed SP fatigue specimens were mounted, ground and polished back to analyse in further detail the mechanisms of crack growth through the microstructure in each material. Figure 9 presents images taken using backscattered electrons of the path of the crack through the two microstructures respectively. The crack behaviour through the EBM microstructure (Figure 9(a)) is complex with the crack travelling in a transgranular manner through the columnar prior $\beta$ grains but the effect of the $\alpha$ laths is less clear. Overall the crack may be deemed to propagate in an interlath manner.

In the forged specimen (Figure 9(b)), the radial crack propagates in a transgranular fashion through the grains 
of the bimodal microstructure for the majority of the crack growth with some intergranular crack growth also present. This is in accordance with the behaviour seen in Figure 10, which shows the crack path through the microstructure of a forged Ti-6Al-4V uniaxial fatigue specimen tested with an $\mathrm{R}$ ratio of -1 . The path of this crack is also largely mixed mode with aspects of both intergranular and transgranular crack propagation mechanisms present.

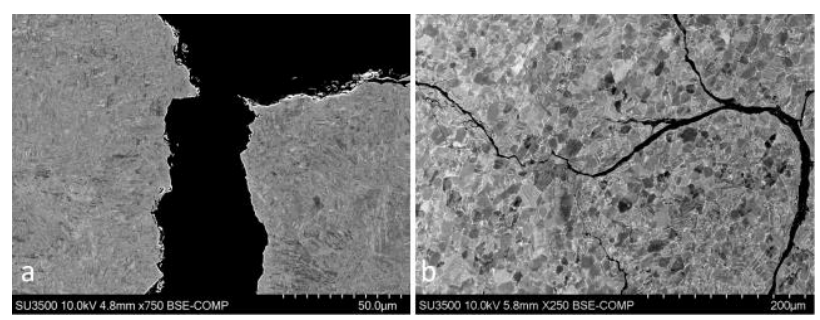

Figure 9. Crack path morphologies for (a) EBM and (b) Forged Ti-6Al-4V SPF test specimens.

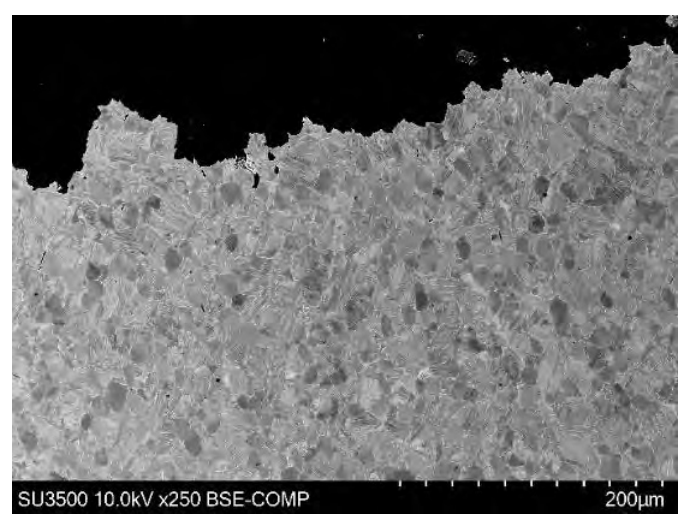

Figure 10. Crack path morphology for a Forged Ti-6Al-4V uniaxial fatigue test.

\section{Conclusions}

This paper details the ongoing research at Swansea University in developing a bespoke SPF testing capability. A series of test results have been presented from novel SPF experiments on two Ti-6Al-4V variants, from which comparative force-number of fatigue cycles to failure curves were determined. The two materials showed a very similar response and the post-test fractography supports this trend. Additional information is generated in the form of representative forcedisplacement fatigue loops which reflect the deformation behaviour of the miniature disc specimen subjected to SP fatigue type loading. An investigation of the crack path morphologies from a SPF test compared to a uniaxial experiment have produced a similar mechanism of failure, signifying that the proposed test method shows real promise for material property evaluation of miniature disc specimens.

\section{Future work}

Further research is in progress with the authors now assessing the effects of a number of fatigue testing parameters including: alternative loading ratios, such as a zero to maximum cycle to investigate whether characteristic fatigue markings are present; loading frequencies to replicate low cycle fatigue conditions; different loading waveforms such as a trapezoid and how this compares to sinusoidal behaviour and also the impact of performing such experiments at elevated temperature and whether the material behaves in a similar manner as would be expected in a uniaxial regime.

Given that the results presented in this paper have been generated under load control conditions, the authors will also investigate the damage evolution of a small punch disc under a displacement controlled cycle, in an attempt to replicate strain-control type fatigue damage.

This additional testing will complement ongoing empirical and numerical modelling efforts to correlate SP fatigue results with uniaxial results and endeavour to understand the stress evolution of a SP disc that is subjected to such a complex form of biaxial loading. A series of interrupted tests will also be performed to aid the understanding of the onset of cracking when subjected to a fatigue type loading condition.

The current research was funded under the EPSRC Rolls-Royce Strategic Partnership in Structural Metallic Systems for Gas Turbines (grants EP/H500383/1 and EP/H022309/1). The provision of materials and supporting information from RollsRoyce plc is gratefully acknowledged by the authors. Mechanical tests were performed at Swansea Materials Research and Testing Ltd. (SMaRT).

\section{References}

[1] S. Tammas-Williams, H. Zhao, F. Léonard, F. Derguti, I. Todd, and P. B. Prangnell, Mater. Charact., 102, 47-61 (2015)

[2] L. E. Murr, E. V Esquivel, S. A. Quinones, S. M. Gaytan, M. I. Lopez, E. Y. Martinez, F. Medina, D. H. Hernandez, E. Martinez, J. L. Martinez, S. W. Stafford, D. K. Brown, T. Hoppe, W. Meyers, U. Lindhe, and R. B. Wicker, Mater. Charact., 60, 2, 96-105 (2009)

[3] M. Bruchhausen, K. Turba, F. De Haan, P. Hähner, T. Austin, and Y. De Carlan, J. Nucl. Mater., 444, 1-3, 283-291 (2014)

[4] S. P. Jeffs, R. J. Lancaster, and T. E. Garcia, Mater. Sci. Eng. A, 636, 529-535 (2015)

[5] S. Davies, S. Jeffs, R. Lancaster, and G. Baxter, "High Temperature Deformation Mechanisms in a DLD Nickel Superalloy," Materials (Basel)., 10, 5, 457 (2017)

[6] T. E. García, C. Rodríguez, F. J. Belzunce, and C. Suárez, J. Alloys Compd., 582, 708-717 (2014)

[7] K. Turba, R. Hurst, and P. Hähner, Int. J. Press. Vessel. Pip., 111-112, 155-161 (2013)

[8] M. Bruchhausen, S. Holmström, I. Simonovski, T. Austin, J. M. Lapetite, S. Ripplinger, and F. de Haan, Theor. Appl. Fract. Mech., 86, 2-10 (2016) [9] D. Andrés, R. Lacalle, and J. A. Álvarez, Mater. Des., 96, 122-130 (2016)

[10] R. J. Lancaster, W. J. Harrison, and G. Norton, Mater. Sci. Eng. A, 626, 263-274 (2015) 
[11] R. C. Hurst, R. J. Lancaster, S. P. Jeffs, and M. R. Bache, Theor. Appl. Fract. Mech., 86, 69-77 (2016)

[12] T. Izaki, T. Kobayashi, J. Kusumoto, and A. Kanaya, Int. J. Press. Vessel. Pip., 86, 9, 637-642 (2009)

[13] X. Gong, T. Anderson, and K. Chou, Manuf. Rev., 1, 2, 1-12 (2014)

[14] N. Hrabe and T. Quinn, Mater. Sci. Eng. A, 573, 271-277 (2013)

[15] CEN Workshop Agreement CWA 15267, CEN Members National Standards Body, 1-69 (2007) 